\title{
Amplitude Correction factors Of Korean VLBi Network OBSERVATIONS ${ }^{\dagger}$
}

\author{
Sang-Sung LeE ${ }^{\ddagger 1,2}$, Do-Young Byun ${ }^{1}$, Chung Sik Oh ${ }^{1}$, Hyo Ryoung Kim ${ }^{1}$, Jongsoo Kim ${ }^{1,2}$, Taehyun Jung ${ }^{1,2}$, \\ Se-Jin OH ${ }^{1}$, Duk-Gyoo RoH ${ }^{1}$, Dong-Kyu Jung ${ }^{1}$, And JAe-Hwan YeOM ${ }^{1}$ \\ ${ }^{1}$ Korea Astronomy and Space Science Institute, 776 Daedeok-daero, Yuseong-gu, Daejeon 34055, Korea;sslee@kasi.re.kr \\ ${ }^{2}$ Korea University of Science and Technology, 217 Gajeong-ro, Yuseong-gu, Daejeon 34113, Korea
}

Received July 9, 2015; accepted September 1, 2015

\begin{abstract}
We report results of investigation of amplitude calibration for very long baseline interferometry (VLBI) observations with Korean VLBI Network (KVN). Amplitude correction factors are estimated based on comparison of KVN observations at $22 \mathrm{GHz}$ correlated by Daejeon hardware correlator and DiFX software correlator in Korea Astronomy and Space Science Institute (KASI) with Very Long Baseline Array (VLBA) observations at $22 \mathrm{GHz}$ by DiFX software correlator in National Radio Astronomy Observatory (NRAO). We used the observations for compact radio sources, 3C 454.3, NRAO 512, OJ 287, BL Lac, 3C 279, 1633+382, and 1510-089, which are almost unresolved for baselines in a range of 350-477 km. Visibility data of the sources obtained with similar baselines at KVN and VLBA are selected, fringe-fitted, calibrated, and compared for their amplitudes. We find that visibility amplitudes of KVN observations should be corrected by factors of 1.10 and 1.35 when correlated by DiFX and Daejeon correlators, respectively. These correction factors are attributed to the combination of two steps of 2-bit quantization in KVN observing systems and characteristics of Daejeon correlator.
\end{abstract}

Key words: Techniques: interferometric — Instrumentation: interferometers - Radio continuum: galaxies

\section{INTRODUCTION}

The Korean VLBI Network (KVN) was built in Korea, as the first mm-dedicated Very Long Baseline Interferometry (VLBI) network, with its main goals for high resolution multi-frequency study of the formation and death of stars, the structure and dynamics of our own Galaxy, and the nature of active galactic nuclei (AGNs) (Lee et al. 2011, 2014). The KVN consists of three 21-m radio telescopes: KVN Yonsei Radio Telescope (KY), KVN Ulsan Radio Telescope (KU), and KVN Tanma Radio Telescope (KT), with baseline lengths of 305-477 km, operating at four frequency bands of $22 \mathrm{GHz}, 43 \mathrm{GHz}, 86 \mathrm{GHz}$, and $129 \mathrm{GHz}$. The maximum angular resolution at the $129 \mathrm{GHz}$ band is 1 milliarcsecond (mas).

KVN observations are amplitude calibrated following a conventional VLBI calibration method and involves the following steps: (a) calibration for amplitude variation due to atmospheric fluctuation, receiver noise fluctuation, elevation dependence of the antenna gains, etc, (b) amplitude correction for the atmospheric opacity on an antenna, and (c) re-normalization of the fringe amplitude for restoring amplitude distortion due to quantization (Lee et al. 2014). For confirmation of the calibration, the calibration of the KVN data correlated using the DiFX correlator is then compared with that of the Very Long Baseline Array (VLBA) obser-

\footnotetext{
Corresponding Author: S.-S. Lee

${ }^{\dagger}$ Part of a special issue on the Korean VLBI Network (KVN)

${ }^{\ddagger}$ sslee@kasi.re.kr
}

vations conducted on the same source and in a similar time, assuming the amplitude calibration of the VLBA has been confirmed. Lee et al. (2014) compared the KVN $22 \mathrm{GHz}$ observations conducted on 2010 October 1 with the VLBA observations on 2010 October 2. They selected the $u v$-data with the same $u v$-distances from both observations for extragalactic compact radio sources, and compared them with each other, estimating the correction factors of $<13 \%$ for individual antennae. Petrov et al. (2012) found that the amplitude correction factors of KVN observations at $43 \mathrm{GHz}$ are within $30 \%$ based on the similar comparison with VLBA observations. However, the authors of the previous works could not identify the sources to which these amplitude correction factors could be attributed, and whether they should be applied to all KVN observations.

In this paper, we report our understanding of the amplitude calibrations of the KVN systems based on observational tests of the requantization losses. We present our findings for the respective correction factors to be applied to KVN data correlated by DiFX and Daejeon correlators. In Section 2, we overview general aspects of the KVN observing systems focusing on the two-step quantization loss. In Section 3, we summarize the observation data used and describe the data reduction procedure. In Section 4, we report the results of the amplitude correction factors. Finally, we discuss our results and present our conclusions in Section 5. 


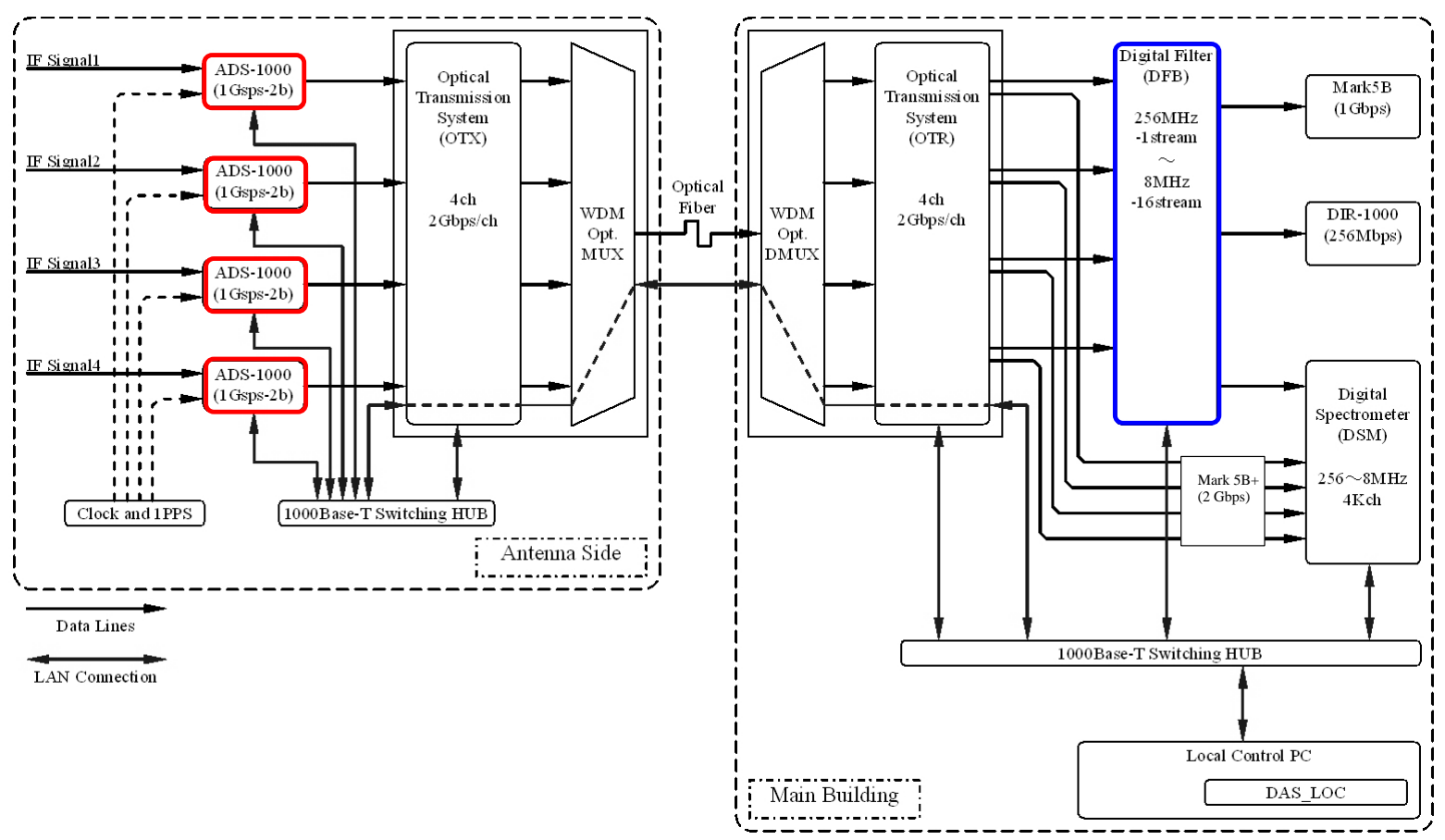

Figure 1. Schematic diagram of KVN Data Acquisition System. Adopted and modified from Oh et al. (2011). The digital samplers and the DFB are outlined in red and blue, respectively.

\section{OVERVIEW OF THE KVN OBSERVING SYSTEMS}

The KVN observing system consists of three 21-m antennae, quasi-optical systems, receivers operating at four frequency bands - $22 \mathrm{GHz}, 43 \mathrm{GHz}, 86 \mathrm{GHz}$, and $129 \mathrm{GHz}$ - and backend systems, as described in Lee et al. (2011). The backend systems include the KVN Data Acquisition System (DAS) which consists of four subsystems: digital samplers, optical transmission system (OTS), digital filter bank (DFB) and digital spectrometer (DSM), as shown in Figure 1. The digital samplers in the antenna cabin digitize the observation data into 2-bit data streams with four quantization levels. The OTS transmits the digitized signals to the observing building through optical fibers. The digitized signals are fed into the digital filter bank (DFB), which divides the input into 16 sub-bands with a frequency interval of $16 \mathrm{MHz}$. The processed signals are recorded by the Mark $5 \mathrm{~B}$ system at an aggregate recording rate of $1024 \mathrm{Mbps}$. For the observations at the aggregate recording rate of $2048 \mathrm{Mbps}$, the digitized signals from the digital samplers can be directly recorded into the Mark 5B+ system with a single band of $512 \mathrm{MHz}$ bandwidth, without passing through the DFB. The recorded data are shipped to the Korea-Japan Correlation Center (KJCC) and correlated with the DiFX software correlator or the Daejeon hardware correlator. In this section, we focus on describing the digital sampler and the digital filter bank systems, which generate quantization loss of the data, although detailed information for the KVN DAS is described in Oh et al. (2011), and on introducing the DiFX software correlator and Daejeon hardware correlator.

\subsection{Digital sampler and Digital filter bank}

In VLBI systems, a digital sampler is preferable to analog sampler due to important practical advantages in compensating time delays and measuring crosscorrelation of signals (Thompson et al. 2001), for example, (a) higher accuracy ( 10-100 psec) of the long delay can be easily obtained in digital signal processing, and (b) the digital signal is not distorted by the digital instrumentation except for quantization which effects can be estimated. The digital sampler samples the voltages of the analog signals at periodic intervals and quantizes the sampled value of the voltages with a small number of bits (e.g., 1-bit or 2-bit). The low level (or small bit number) quantization results in a quantization noise, i.e., a loss of sensitivity in digitizing the analog signals. Therefore, an optimal number of quantization levels is important in the digital signal process, and the investigations of the optimal quantization levels can be found in the literature (Thompson et al. 2001, and references therein). Thees investigations result in well-known efficiency factors for Nyquist sampling, 0.637 for 2-level quantization, 0.810 for 3level, and 0.881 for 4 -level. It should be noted that the efficiency factors for 3-level and 4-level quantizations are the maximum values when the sampling threshold is properly chosen, and hence, in practice, lower efficiencies are expected if the sampling threshold is not properly adjusted.

The digital samplers used in KVN is based on ADS1000 Giga-Bit-Sampler (GBS) developed by the National Institute of Information and Communications Technology (NiCT), Japan (Nakajima et al. 2000). The 
digital sampler quantizes an intermediate frequency analog signal, converted from the radio frequency signals by receivers, with a bandwidth of $512 \mathrm{MHz}$ and in four quantization levels (or in two bits), in order to achieve a higher sensitivity in the quantization. Since the bandwidth of the sampled signals by the digital sampler is $512 \mathrm{MHz}$, it is necessary to process the signals fitted into a bandwidth of $256 \mathrm{MHz}$ for the Mark $5 \mathrm{~B}$ system. Moreover, it is also required to channelize the whole frequency band into sub-bands and pick up the desired sub-bands optimized for scientific interests. Therefore, the DFB is introduced in the KVN DAS for flexibly channelizing and extracting the desired frequency bands according to the scientific purposes. In fact, the DFB filters out the desired frequency bands in the digital wave-forms generated by the digital samplers with four-level (2-bit) quantized sampling. Since the data processing rate is limited by the instrumental capability, it is required to re-quantize the signal after the digital filtering (Iguchi et al. 2005).

\subsection{DiFX and Daejeon correlator}

A software correlator based on DiFX (Distributed FX; Deller et al. 2007, 2011) has been installed and used mainly for correlating data from the KVN observations. The software version of DiFX has been upgraded and the current version is 2.3. Detailed information of the DiFX installed for the KVN is described in Lee et al. (2014). A new hardware correlator (Daejeon correlator) was developed in 2009 by Korea Astronomy and Space Science Institute (KASI) and National Astronomical Observatory of Japan (NAOJ). The Daejeon correlator is mainly used for the East Asian VLBI Network (EAVN) consisting of Korean VLBI Network (KVN), Japanese VLBI Network (JVN) including the VLBI Exploration of Radio Astrometry (VERA) in Japan, and the Chinese VLBI Network (CVN). More information of the Daejeon correlator is summarized in Lee et al. (2014).

In order to evaluate the performance of the new hardware correlator, Lee et al. (2015) carefully compared the correlation outputs of the DiFX and Daejeon correlators, using the KVN observations conducted at $22 \mathrm{GHz}$ on 2011 January 28-29. They found that the two correlators are comparable to each other in the correlation outputs except for the visibility amplitudes of the hardware correlator being lower by a factor of $<8 \%$ than those of the DiFX correlator. The amplitude difference is due to the characteristics of the hardware correlator: (a) the way of fringe phase tracking (causing an amplitude loss of $\sim 5 \%$ due to coarse phase tracking and see Iguchi et al. (2000)) and (b) an unusual pattern of the amplitude of the correlation output (causing an amplitude loss of $\sim 3 \%$ ). Fortunately, it was announced by the KJCC operation team that the latter characteristic has been fixed for the data correlated after 2015 March.

It should be noted that Lee et al. (2015) did not apply digital correction (by switching off the option DIGICOR of the task FITLD) in order to compare the raw outputs from the two correlators. In fact, the op-

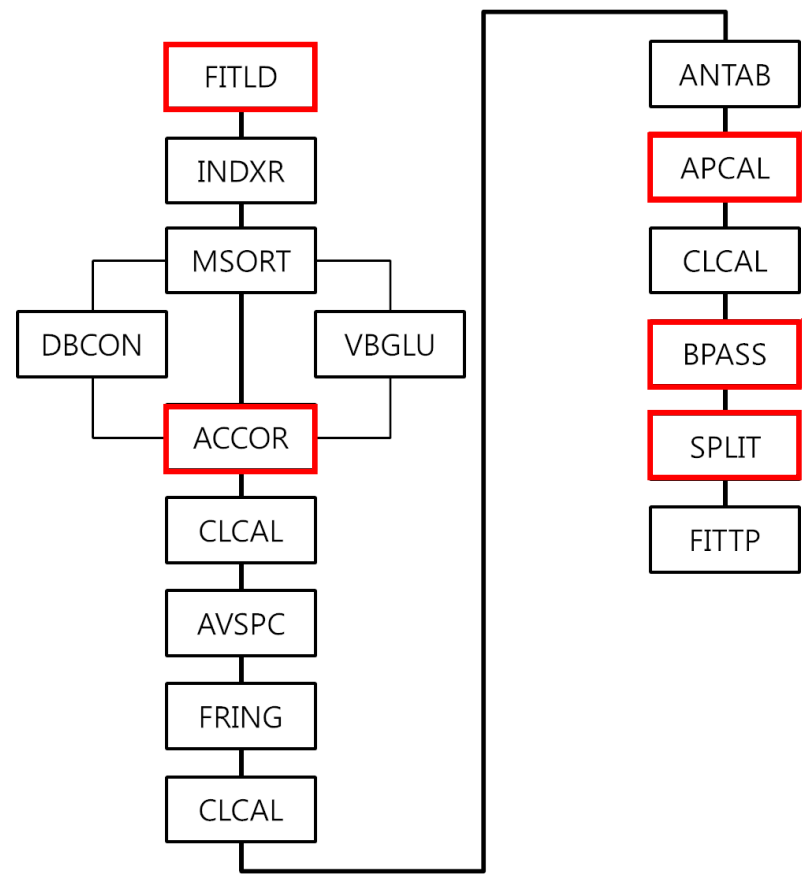

Figure 2. Schematic diagram of VLBI data reduction with AIPS. Adopted and modified from Lee et al. (2015). The AIPS Tasks related with amplitude calibration are outlined in red.

tion is usually required for proper amplitude calibration in case of DiFX data, unless DiFX was configured to use Tsys files during correlation. However, for the case of the hardware correlator, the option DIGICOR does not work properly. It may be because the Daejeon correlator generates FITS data whose header information is not optimized for the use of the option DIGICOR. Since the scaling difference between DIGICOR $\mathrm{ON} / \mathrm{OFF}$ cases could be a few percent (e.g., 1/0.88 for 2-bit quantization), the actual difference of the amplitudes between KJCC and DiFX after applying the option DIGICOR is larger than $8 \%$. In order to investigate the difference, it is required to compare the DIGICOR-ON-outputs of the KVN data correlated by DiFX and Daejeon correlator with those of the VLBA data correlated by DiFX.

\section{OBSERVATIONAL DATA AND DATA REDUCTION}

\subsection{Observational data}

We compared KVN data obtained at $22 \mathrm{GHz}$ with VLBA data at $22 \mathrm{GHz}$. We selected the VLBA data obtained close to the KVN data in time within a month in order to reduce source variability effect. Observational data at $22 \mathrm{GHz}$ are used since we expect relatively less uncertainty of the amplitude calibration at this frequency than at higher frequencies, due to the atmospheric effect, pointing offset, etc. We used two KVN experiments (r11027 and n14sl01h) on 2011 January 28 and 2014 April 22 together with four VLBA experiments (BF104A, BM272, TD074, and S6096A) on 2011 January 17, 2011 February 26, 2014 April 13 
Table 1

Log of observational data

\begin{tabular}{lcccccccc}
\hline $\begin{array}{l}\text { VLBI } \\
\text { Network } \\
(1)\end{array}$ & $\begin{array}{c}\text { Experiment } \\
\text { Name }\end{array}$ & $\begin{array}{c}\text { Experiment } \\
\text { Date } \\
(2)\end{array}$ & $\begin{array}{c}\text { Frequency Band } \\
(\mathrm{GHz})\end{array}$ & $\begin{array}{c}\text { Bit rate } \\
(\mathrm{bit})\end{array}$ & $\begin{array}{c}\text { Frequcny } \\
\text { Channels } \\
(5)\end{array}$ & $\begin{array}{c}\text { Sampling } \\
\left(\mathrm{Mbit} \mathrm{s}^{-1}\right)\end{array}$ & $\begin{array}{c}\text { Bandwidth } \\
(\mathrm{MHz}) \\
(7)\end{array}$ & $\begin{array}{c}\text { Polarization } \\
(9)\end{array}$ \\
\hline VLBA & bf104a & 2011 Jan 17 & $23.50-23.98$ & 2 & 8 & 256 & 64 & LCP \\
KVN & r11027b & 2011 Jan 28 & $22.00-22.45$ & 2 & 16 & 1024 & 256 & LCP \\
VLBA & bm272 & 2011 Feb 26 & $22.22-22.24$ & 2 & 4 & 128 & 32 & Dual \\
VLBA & td074 & 2014 Apr 13 & $22.01-22.11$ & 2 & 4 & 512 & 128 & Dual \\
KVN & n14sl01h & 2014 Apr 22 & $21.70-21.76$ & 2 & 4 & 1024 & 64 & LCP \\
VLBA & s6096a & 2014 Apr 26 & $22.09-22.36$ & 2 & 8 & 1024 & 256 & Dual \\
\hline
\end{tabular}

and 2014 April 26. All experiments were conducted at $22 \mathrm{GHz}$ bands. The KVN and VLBA data for this paper are summarized in Table 1.

\subsection{Data reduction}

For all of the data sets selected, we performed the same post-correlation processing using the NRAO Astronomical Imaging Processing System (AIPS), following a standard processing procedure as described in Figure 2. The correlated output FITS data was uploaded by FITLD task. In using FITLD, we did apply digital correction $($ DIGICOR $=1$ ) for all data sets whether it works or not. This is an opportunity to correct for the quantization loss due to the digital samplers. As described in Section 2.2, for the VLBI data (e.g., the KVN and VLBA data) correlated by DiFX, the quantization loss can be corrected by the DIGICOR option, whereas, for the data correlated by the Daejeon correlator, the DIGICOR option does not change the visibility amplitude, hence no correction for the quantization loss. The loaded data was indexed with INDXR task, sorted by time and baseline by MSORT, combined, if necessary, in time or frequency by DBCON or VBGLU, respectively, and corrected by ACCOR for amplitude errors in the digital sampling. This is second step of amplitude calibration. After applying the correction information from ACCOR by CLCAL and fringe-fitting with FRING, atmospheric opacity change and amplitude errors due to the atmospheric fluctuation, are corrected by APCAL. System noise temperatures and antenna gains measured at each observatory are used for converting the correlation coefficient to sky brightness and correcting for the amplitude errors. Depending on weather conditions and the accuracy of antenna gain measurements, we may expect amplitude calibration errors as large as 10-30\% for any VLBI array. The amplitude-calibrated data are further corrected for effect of bandpass filter on the spectrum shape using BPASS task. We used the full bandwidth for the amplitude calibration in BPASS. For multiplesource data, we split the data for individual source by SPLIT with chopping $10 \%$ of frequency channels at the band edge. This step is necessary for amplitude calibration since the excluded channels may be suffering from the amplitude loss due to the uncorrected effect of the bandpass filter.

\subsection{Amplitude correction}

Any unwanted visibility amplitude offset, which we want to correct, can be corrected in conducting the APCAL. The specific APCAL options and parameters for the amplitude correction are (a) $\operatorname{APARM}(1)=B$, a correction factor (e.g., 1.10), which is called B factor in AIPS, (b) OPCODE $=$ ', and (c) DOFIT $>1$. The APCAL with this setup corrects the amplitude of the visibility by multiplying the original amplitude with the correction factor, $B$. It should be noted that since the APCAL corrects the amplitude of every visibility before the time and frequency averaging, the resultant scatter of the visibility amplitude will also increase by a factor of $<B$.

\section{RESULTS}

After the amplitude calibration, we selected visibility data of 3C 454.3, NRAO 512, OJ 287, BL Lac, 3C 279, $1633+382$, and 1510-089, obtained with similar baselines at KVN and VLBA. Since, at $22 \mathrm{GHz}$ band, the KVN baseline lengths are in the range of 20-35 M $\lambda$, we selected the VLBA data on the baselines, Fort Davis (FD) to Pie Town (PT), Kitt Peak (KP) to PT, Los Alamos (LA) to PT, FD to LA, FD to KP, or KP to Owens Valley (OV), whose baseline lengths are in the range of 17-35 $\mathrm{M} \lambda$. The visibility data were first averaged in time at an interval of $30 \mathrm{~s}$. The amplitudes of the visibility data for each baseline were averaged for all IF bands. The estimated mean visibility amplitudes for all baselines and sources are summarized in Table 2. The estimated mean amplitudes are compared between the KVN and VLBA baselines with similar baseline lengths and also compared between the KVNDiFX and the KVN-Dajeon data. By taking into the comparison results, we determined the amplitude correction factors for the KVN observations correlated by DiFX and Daejeon correlators.

In the KVN baselines (or resolutions), the source compactness (defined as the ratio of the correlated flux density on the longest baseline to that on the shortest baseline) is close to unity, implying that the source is very compact or unresolved. Since some of the VLBA baselines selected are not aligned with the KVN baselines in the position angle (P.A.) of the baseline, we may expect that source structure may result in different correlated flux density on KVN baselines depending on the 
Table 2

Mean visibility amplitude

\begin{tabular}{|c|c|c|c|c|c|c|c|c|}
\hline $\begin{array}{l}\text { Source } \\
\text { (1) }\end{array}$ & $\begin{array}{c}\text { Telescope } \\
\text { (2) }\end{array}$ & $\begin{array}{c}\text { Correlator } \\
\text { (3) }\end{array}$ & $\begin{array}{l}\text { Epoch } \\
(4)\end{array}$ & $\begin{array}{l}\text { Baseline } \\
(5)\end{array}$ & $\begin{array}{l}\text { Length } \\
(\mathrm{M} \lambda) \\
(6)\end{array}$ & $\begin{array}{l}\text { P.A. } \\
\left(\begin{array}{l}\circ \\
(7)\end{array}\right. \\
(7)\end{array}$ & $\begin{array}{l}\text { Amplitude } \\
(\mathrm{Jy}) \\
(8)\end{array}$ & $\begin{array}{c}\text { Corrected amplitude } \\
\text { (Jy) } \\
(9)\end{array}$ \\
\hline \multirow{14}{*}{ 3C 454.3} & \multirow[t]{3}{*}{ KVN } & \multirow{3}{*}{ DiFX } & \multirow{3}{*}{$2011 \operatorname{Jan} 28$} & KT-KY & 34 & 20 & $24.7 \pm 0.7$ & $27.1 \pm 0.7$ \\
\hline & & & & KT-KU & 26 & 55 & $23.8 \pm 0.7$ & $26.1 \pm 0.8$ \\
\hline & & & & KU-KY & 20 & -30 & $25.6 \pm 0.4$ & $28.2 \pm 0.5$ \\
\hline & \multirow[t]{3}{*}{ KVN } & \multirow[t]{3}{*}{ Daejeon } & \multirow[t]{3}{*}{2011 Jan 28} & KT-KY & 34 & 20 & $20.2 \pm 0.7$ & $27.3 \pm 1.0$ \\
\hline & & & & KT-KU & 26 & 55 & $19.5 \pm 0.7$ & $26.4 \pm 0.9$ \\
\hline & & & & KU-KY & 20 & -30 & $21.1 \pm 0.6$ & $28.4 \pm 0.7$ \\
\hline & \multirow[t]{3}{*}{ VLBA } & \multirow{3}{*}{ DiFX } & \multirow{3}{*}{2011 Feb 26} & FD-PT & 35 & 20 & $28.2 \pm 0.4$ & - \\
\hline & & & & KP-PT & 28 & 65 & $26.8 \pm 0.8$ & - \\
\hline & & & & LA-PT & 17 & 60 & $28.0 \pm 0.4$ & - \\
\hline & \multirow[t]{2}{*}{$\mathrm{KVN}$} & \multirow[t]{2}{*}{ DiFX } & \multirow[t]{2}{*}{2014 Apr 22} & KU-KY & 19 & 20 & $9.64 \pm 0.06$ & $10.6 \pm 0.06$ \\
\hline & & & & KT-KY & 32 & -20 & $10.4 \pm 0.08$ & $11.4 \pm 0.03$ \\
\hline & \multirow[t]{3}{*}{ VLBA } & \multirow[t]{3}{*}{ DiFX } & 2014 Apr 13 & LA-PT & 16 & 40 & $10.4 \pm 0.08$ & - \\
\hline & & & \multirow[t]{2}{*}{2014 Apr 26} & LA-PT & 18 & 50 & $11.7 \pm 0.02$ & - \\
\hline & & & & KP-PT & 31 & 55 & $11.2 \pm 0.05$ & - \\
\hline \multirow[t]{10}{*}{ NRAO 512} & \multirow[t]{3}{*}{$\mathrm{KVN}$} & \multirow[t]{3}{*}{ DiFX } & \multirow[t]{3}{*}{2011 Jan 28} & KT-KY & 32 & 40 & $0.98 \pm 0.05$ & $1.08 \pm 0.06$ \\
\hline & & & & KU-KY & 22 & 10 & $1.00 \pm 0.05$ & $1.10 \pm 0.05$ \\
\hline & & & & KT-KU & 18 & 80 & $1.00 \pm 0.06$ & $1.10 \pm 0.07$ \\
\hline & KVN & Daejeon & 2011 Jan 28 & KT-KY & 32 & 40 & $0.79 \pm 0.05$ & $1.07 \pm 0.07$ \\
\hline & & & & KU-KY & 22 & 10 & $0.81 \pm 0.05$ & $1.09 \pm 0.06$ \\
\hline & & & & KT-KU & 18 & 80 & $0.80 \pm 0.05$ & $1.08 \pm 0.07$ \\
\hline & VLBA & DiFX & 2011 Jan 17 & KP-PT & 32 & -15 & $1.00 \pm 0.14$ & - \\
\hline & & & & FD-LA & 32 & -55 & $1.11 \pm 0.11$ & - \\
\hline & & & & FD-KP & 31 & 25 & $1.06 \pm 0.13$ & - \\
\hline & & & & LA-PT & 18 & -20 & $1.07 \pm 0.14$ & - \\
\hline OJ 287 & $\mathrm{KVN}$ & DiFX & 2014 Apr 22 & KU-KY & 18 & 5 & $3.74 \pm 0.01$ & $4.08 \pm 0.01$ \\
\hline & & & & KT-KY & 33 & 0 & $4.00 \pm 0.01$ & $4.40 \pm 0.01$ \\
\hline & VLBA & DiFX & 2014 Apr 26 & LA-PT & 18 & 50 & $4.43 \pm 0.01$ & - \\
\hline & & & & KP-PT & 31 & 55 & $4.22 \pm 0.02$ & - \\
\hline BL Lac & $\mathrm{KVN}$ & DiFX & 2014 Apr 22 & KU-KY & 14 & -80 & $4.98 \pm 0.01$ & $5.48 \pm 0.01$ \\
\hline & & & & KU-KY & 17 & -75 & $4.83 \pm 0.03$ & $5.32 \pm 0.03$ \\
\hline & & & & KT-KY & 33 & -35 & $4.31 \pm 0.01$ & $4.54 \pm 0.02$ \\
\hline & VLBA & DiFX & 2014 Apr 26 & LA-PT & 14 & 75 & $5.72 \pm 0.01$ & - \\
\hline & & & & LA-PT & 17 & 55 & $5.27 \pm 0.01$ & - \\
\hline & & & & KP-PT & 31 & 45 & $4.50 \pm 0.02$ & - \\
\hline $3 \mathrm{C} 279$ & KVN & DiFX & 2014 Apr 22 & KU-KY & 12 & -20 & $24.6 \pm 0.07$ & $27.1 \pm 0.08$ \\
\hline & & & & KT-KU & 26 & 55 & $23.3 \pm 0.03$ & $25.6 \pm 0.03$ \\
\hline & & & & KT-KY & 28 & 20 & $23.6 \pm 0.02$ & $26.0 \pm 0.02$ \\
\hline & VLBA & DiFX & 2014 Apr 26 & LA-PT & 12 & 45 & $26.0 \pm 0.07$ & - \\
\hline & & & & KP-PT & 26 & 55 & $26.3 \pm 0.2$ & - \\
\hline & & & & KP-PT & 28 & 45 & $24.7 \pm 0.1$ & - \\
\hline $1633+382$ & $\mathrm{KVN}$ & DiFX & 2014 Apr 22 & KU-KY & 17 & -70 & $2.70 \pm 0.01$ & $2.98 \pm 0.02$ \\
\hline & & & & KT-KY & 33 & -35 & $2.61 \pm 0.02$ & $2.88 \pm 0.02$ \\
\hline & VLBA & DiFX & 2014 Apr 26 & LA-PT & 17 & 30 & $2.99 \pm 0.01$ & - \\
\hline & & & & KP-LA & 33 & 80 & $2.61 \pm 0.02$ & - \\
\hline $1510-089$ & $\mathrm{KVN}$ & DiFX & 2014 Apr 22 & KT-KU & 17 & 45 & $3.11 \pm 0.01$ & $3.42 \pm 0.01$ \\
\hline & & & & KT-KY & 27 & -20 & $3.20 \pm 0.02$ & $3.52 \pm 0.02$ \\
\hline & VLBA & DiFX & 2014 Apr 26 & LA-PT & 17 & 55 & $3.33 \pm 0.01$ & - \\
\hline & & & & KP-OV & 27 & -15 & $3.48 \pm 0.04$ & - \\
\hline
\end{tabular}

position angle of the baselines. However, we expect that source structure effects are very small due to the compact structure of the sources. We find that, on average, the visibility amplitudes of VLBA data are higher than those of KVN-DiFX data by $10 \%$ and much higher than those of KVN-Daejeon data by $35 \%$, as shown in Ta- ble 3 . This is because the visibility amplitudes of the KVN-DiFX data are higher than those of the KVNDaejeon by $23 \%$.

Using the amplitude correction factors determined, we corrected the amplitude of the KVN observations according to Section 3.3. As shown in Table 2 (Column 
Table 3

Comparison of the visibility amplitude

\begin{tabular}{|c|c|c|c|c|c|}
\hline Source & Epoch & Baseline & $\frac{S_{\mathrm{VLBA}}}{S_{\mathrm{KVN}, \mathrm{DiFX}}}$ & $\frac{S_{\mathrm{VLBA}}}{S_{\mathrm{KVN}, \mathrm{Daej}}}$ & $\frac{S_{\mathrm{KVN}, \mathrm{DiFX}}}{S_{\mathrm{KVN}, \mathrm{Daej}}}$ \\
\hline \multirow{5}{*}{ 3C 454.3} & 2011 Jan 28 & KT-KY & 1.14 & 1.40 & 1.22 \\
\hline & & KU-KY & 1.13 & 1.37 & 1.22 \\
\hline & & KT-KU & 1.09 & 1.33 & 1.21 \\
\hline & 2014 Apr 22 & KT-KY & 1.08 & - & - \\
\hline & & KU-KY & 1.09 & - & - \\
\hline \multirow[t]{3}{*}{ NRAO 512} & 2011 Jan 28 & KT-KY & 1.08 & 1.34 & 1.24 \\
\hline & & KU-KY & - & - & 1.23 \\
\hline & & KT-KU & 1.07 & 1.34 & 1.25 \\
\hline \multirow{2}{*}{ OJ 287} & 2014 Apr 22 & KT-KY & 1.06 & - & - \\
\hline & & KU-KY & 1.19 & - & - \\
\hline \multirow[t]{2}{*}{ BL Lac } & 2014 Apr 22 & KT-KY & 1.04 & - & - \\
\hline & & KU-KY & 1.12 & - & - \\
\hline \multirow[t]{3}{*}{$3 \mathrm{C} 279$} & 2014 Apr 22 & KT-KY & 1.05 & - & - \\
\hline & & KU-KY & 1.06 & - & - \\
\hline & & KT-KU & 1.13 & - & - \\
\hline \multirow[t]{2}{*}{$1633+382$} & 2014 Apr 22 & KT-KY & 1.14 & - & - \\
\hline & & KU-KY & 1.11 & - & - \\
\hline \multirow[t]{2}{*}{$1510-089$} & 2014 Apr 22 & KT-KY & 1.09 & - & - \\
\hline & & KU-KY & 1.08 & - & - \\
\hline Mean & & & 1.10 & 1.35 & 1.23 \\
\hline
\end{tabular}

Table 4

Amplitude correction factors

\begin{tabular}{lcccc}
\hline Telescope & Correlator & Frequency band & Correction Factor & Remarks \\
\hline KVN & DiFX & $22 \mathrm{GHz}$ & 1.10 & \\
& & $43-129 \mathrm{GHz}$ & 1.10 & \\
& Daejeon & $22 \mathrm{GHz}$ & 1.35 & before 2015 March \\
& & $22 \mathrm{GHz}$ & 1.30 & after 2015 March \\
& & $43-129 \mathrm{GHz}$ & 1.35 & before 2015 March \\
& & $43-129 \mathrm{GHz}$ & 1.30 & after 2015 March \\
VERA & DiFX & $22-43 \mathrm{GHz}$ & 1.10 & \\
& Daejeon & $22-43 \mathrm{GHz}$ & 1.35 & before 2015 March \\
& & $22-43 \mathrm{GHz}$ & 1.30 & after 2015 March \\
\hline
\end{tabular}

The correction factors should be applied only to the KVN observations at a recording rate of 1024 Mbps. The correction factors in italic are the suggested values.

8), the mean visibility amplitudes for the KVN data are corrected to be consistent with the VLBA data. The scatter in the visibility amplitude increases by the applied factors as expected.

\section{Discussion}

The difference of the calibrated visibility amplitudes between KVN-DiFX, KVN-Daejeon, and VLBA-DiFX data are attributed to several aspects. We will consider here the factors which are important in causing the amplitude difference, and determine the correction factors for the KVN amplitude calibration.

\subsection{Quantization loss}

The quantization loss in digitizing analog signals has been theoretically analyzed (e.g., Thompson et al.
2001), and the digital loss in re-quantizing the digitized signals has been numerically investigated by Iguchi et al. (2005). The quantization loss is known to be 0.88 for the four-level (2-bit) quantization and the accumulated digital loss was shown to be $0.81(=0.88 \times 0.92)$ in four-level re-quantization of the four-level quantized signals. Thus the additional digital loss due only to the four-level re-quantization is 0.92 in the case of the input signals of four-level quantization.

KVN observations experience a four-level quantization in the digital samplers and an additional four-level re-quantization in the DFB. As described in Section 2.1, digital sampling and filtering are based on four-level (2bit) quantization, resulting in an accumulated digital loss factor of 0.81 . In a standard calibration procedure, for example as described in Section 3.2, a quantization loss factor of 0.88 is corrected by switching on the op- 
tion DIGICOR of the FITLD task. Thus, the quantization loss due to the digital samplers can be corrected with the standard calibration procedure, whereas the re-quantization loss by the DFB is not corrected, following general procedure. Consequently, the remaining amplitude loss factor for the properly calibrated visibility of the DiFX correlator is about 0.92 , and the correction factor is 1.09 , which is similar to the amplitude correction factor $\frac{S_{\mathrm{VLBA}}}{S_{\mathrm{KVN}, \mathrm{DiFx}}}=1.10$. The difference between the two correction factors, 1.09 and 1.10, may arise from the difference of the amplitude calibration uncertainties of KVN and VLBA (see below).

\subsection{Characteristics of Daejeon correlator}

As reported by Lee et al. (2015), the calibrated visibility of the Daejeon correlator suffers from two amplitude loss factors: (a) $0.95(5 \%)$ due to the fringe tracking scheme and (b) 0.97 (3\%) due to the double-layer pattern problem. The fringe tracking scheme is the characteristics of the VLBI Correlation System (VCS) of the hardware correlator, which should be a constant loss factor as long as the hardware correlator uses the same fringe tracking scheme in the VCS. The doublelayer pattern problem occurs in serializing the data and mapping the memory in the VCS, and has been solved in correlations after 2015 March (S. J. Oh, priv. communication).

In addition to these loss factors, the calibrated visibility of the Daejeon correlator may experience additional amplitude loss after the proper amplitude calibration since the quantization and re-quantization loss from the KVN observations is not properly corrected using a standard VLBI data reduction procedure, as described in Section 2.2. This characteristics results in an amplitude loss factor of 0.81. Therefore, the total amplitude loss factor for the properly calibrated visibility of the Daejeon correlator is about $0.75(=0.81 \times 0.95 \times 0.97)$, and the correction factor is 1.34 , which is similar to the amplitude correction factor $\frac{S_{\mathrm{VLBA}}}{S_{\mathrm{KVN}, \mathrm{Dajj}}}=1.35$. The difference between the two correction factors, 1.34 and 1.35, may be due to the difference in the amplitude calibration uncertainties of KVN and VLBA (see below). Moreover, the amplitude ratio $\frac{S_{\mathrm{KVN}, \mathrm{DiFx}}}{S_{\mathrm{KVN}, \mathrm{Daej}}}=1.23$ can be explained since the total amplitude loss factor of the Daejeon correlator compared to the DiFX is about 0.81 $(=0.88 \times 0.92)$ and hence the correction factor is 1.23 .

\subsection{Calibration uncertainty and source variability}

In addition to the two factors, the amplitude difference may also be attributed to amplitude calibration uncertainties of KVN and VLBA and to the time-variability of the source brightness and structure. The difference in the uncertainties may depend on the observing frequency band and the weather condition. The source variability effect may be reduced by using contemporaneous data from KVN and VLBA. Therefore, these two factors contribute little to the difference of the visibility amplitude between KVN and VLBA data than the factors described earlier. Caution should be taken here, since the weather conditions are very different in KVN and VLBA, and thus any systematics which could be caused by the weather may not be negligible.

\subsection{Amplitude correction factors}

Since the differences of the calibrated visibility amplitudes between KVN and VLBA data are attributed mainly to the digital characteristics of the KVN VLBI system and the hardware and software characteristics of Daejeon correlator, we may expect that the nature of the difference should remain constant in time and frequency bands. Therefore, one may apply the amplitude correction factors to the future KVN observations at $22 \mathrm{GHz}, 43 \mathrm{GHz}, 86 \mathrm{GHz}$ and $129 \mathrm{GHz}$ bands. The time and frequency dependence of the amplitude correction factors will be extensively investigated by using the KVN data of a KVN key science program, iMOGABA (Interferometric Monitoring of Gamma-ray Bright AGNs) (Algaba et al. 2015, Lee et al. in prep.), and the results will be reported elsewhere.

Our investigations are based on KVN observations. However, the results may be applied to VERA observations, since the backend system (in particular, digital sampler and DFB), of VERA is similar to that of KVN. Thus, we suggest that the amplitude correction factors determined in this paper can be applied to the VERA or KVN-VERA observations. Again, the amplitude correction factors for the KVN-VERA data will be confirmed and reported elsewhere (Oh. et al. in prep.).

Finally, we suggest the use of an amplitude correction factor of $1.30\left(=\frac{1}{0.81 \times 0.95}\right)$ for the VLBI data correlated by the Daejeon correlator since 2015 March, because the double-layer pattern problem has been resolved for the recent correlations. The suggested correction factors are summarized in Table 4 . The correction factors should be applied only to the KVN observations at a recording rate of $1024 \mathrm{Mbps}$.

\section{Conclusion}

The basic assumption that the VLBA amplitude calibration is robust enables to evaluate the amplitude calibration of KVN observations based on the rigorous comparison between the KVN and VLBA data obtained contemporaneously for extragalactic compact radio sources. We find that the VLBA data have higher amplitude than the KVN data by factors of 1.10 and 1.35 for the cases of DiFX and Daejeon correlators. Among several aspects contributing to the amplitude difference, we find that the quantization losses due to the digital samplers and the DFB, and the characteristics of the Daejeon correlator are the main contributors. We expect that the nature of the amplitude losses is constant in time and frequency band. Therefore, we should apply the amplitude correction factors of 1.10 and 1.35 to the future KVN observations correlated by DiFX and Daejeon correlators, respectively. It is also suggested to apply these correction factors to observations using the KVN and VERA combined array. 


\section{ACKNOWLEDgments}

We would like to thank the anonymous referee for important comments and suggestions which have enormously improved the manuscript. We are grateful to all staff members in KVN who helped to operate the array and to correlate the data. The KVN is a facility operated by the Korea Astronomy and Space Science Institute. The KVN operations are supported by KREONET (Korea Research Environment Open NETwork) which is managed and operated by KISTI (Korea Institute of Science and Technology Information). The VLBA is an instrument of the National Radio Astronomy Observatory, which is a facility of the National Science Foundation operated under cooperative agreement by Associated Universities, Inc.

\section{REFERENCES}

Algaba, J. C., Zhao, G. Y., Lee, S.-S., et al. 2015, Interferometric Monitoring of Gamma-ray Bright Active Galactic Nuclei I: Performance Evaluation of Imaging Quality, JKAS, 48, 237

Deller, A. T., Tingay, S. J., Bailes, M., et al. 2017, DiFX: A Software Correlator for Very Long Baseline Interferometry Using Multiprocessor Computing Environments, PASP, 119, 318

Deller, A. T., Brisken, W. F., Phillips, C. J., et al. 2011, DiFX-2: A More Flexible, Efficient, Robust, and Powerful Software Correlator, PASP, 123, 275
Iguchi, S., Kawaguchi, N., Murata, Y., et al. 2000, Development and Performance of the Real-Time VLBI Correlator (RVC), IEICE Trans. Commun., E83-B, 2527

Iguchi, S., Kurayama, T., Kawaguchi, N., \& Kawakami, K. 2005, Gigabit Digital Filter Bank: Digital Backend Subsystem in the VERA Data-Acquisition System, PASJ, 57, 259

Lee, S.-S., Byun, D.-Y., Oh, C. S., et al. 2011, Single-Dish Performance of KVN 21 m Radio Telescopes: Simultaneous Observations at 22 and $43 \mathrm{GHz}$, PASP, 123, 1398

Lee, S.-S., Petrov, L., Byun, D.-Y., et al. 2014, Early Science with the Korean VLBI Network: Evaluation of System Performance, AJ, 147, 77

Lee, S.-S., Oh, C. S., Roh, D.-G., et al. 2015, A New Hardware Correlator in Korea: Performance Evaluation Using KVN Observations, JKAS, 48, 125

Nakajima, J., Sekido, M., \& Suzuyama, T. 2000, Another giga-bit sampler prototype, Nearly completion: ADS1000 development status, IVS CRL-TDC News, 17, 18

Oh, S.-J., Roh, D.-G., Wajima, K., et al. 2011, Design and Development of a High-Speed Data-Acquisition System for the Korean VLBI Network, PASJ, 63, 1229

Petrov, L., Lee, S. S., Kim, J., et al. 2012, Early Science with the Korean VLBI Network: The QCAL-1 $43 \mathrm{GHz}$ Calibrator Survey, AJ, 144, 150

Thompson, A. R., Moran, J. M., \& Swenson, G. W. Jr., 2001, Interferometry and Synthesis in Radio Astronomy, 2nd Edition, (New York: John Wiley \& Sons), 260 